\title{
Magnetic Coupler Robust Optimization Design for Electric Vehicle Wireless Charger Based on Improved Simulated Annealing Algorithm
}

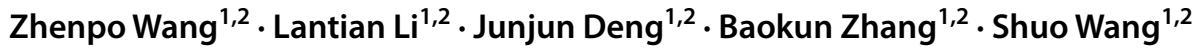

Received: 1 June 2021 / Accepted: 11 November 2021 / Published online: 19 January 2022

(c) The Author(s) 2022

\begin{abstract}
Fleets of autonomous vehicles including shuttle buses, freight trucks, and road sweepers will be deployed in the Olympic Village during Beijing 2022 Winter Olympics. This requires intelligent charging infrastructure based on wireless power transfer technology to be equipped. To increase the misalignment tolerance of a high-power wireless charger, the robustness of the magnetic coupler should be optimized. This paper presents a new type of unipolar coupler, which is composed of three connected coils in series. The dimensional configuration of the coils is analyzed by the finite element method. The characteristic parameters of the coil are identified with their influence on the self-inductance and coupling coefficient. An expert model is built, whose feasibility can be verified in the aimed design domain. Combined with the expert model, an improved simulated annealing algorithm with a backtracking mechanism is proposed. The primary coil can reach the expected characteristics from any starting parameter combination through the proposed optimization algorithm. Under the same conditions in terms of external circuit parameters, ferrite usage, and aluminum shielding, the offset sensitivity of the magnetic coupler can be reduced from $58.79 \%$ to $18.89 \%$. A prototype is established, validating the feasibility of the proposed coil structure with the optimized parameter algorithm.
\end{abstract}

Keywords Wireless power transfer $\cdot$ Magnetic coupler $\cdot$ Simulated annealing algorithm $\cdot$ Robust optimization

\begin{tabular}{ll}
\multicolumn{2}{l}{ Abbreviations } \\
BMS & Battery management system \\
DD Coil & Double-D coil \\
DDQ Coil & Double-D-Quadrature coil \\
FEA & Finite element analysis \\
IPT & Inductive power transfer \\
LCC & Inductor-capacitor-capacitor \\
LCC-LCC & Double-sided LCC \\
LCC-S & LCC-series \\
MS Coil & Multiple series unipolar coil \\
PFC & Power factor correction \\
SA & Simulated annealing \\
S-S & Series-series \\
WPT & Wireless power transfer \\
ZPA & Zero phase angle \\
ZVS & Zero voltage switching
\end{tabular}

Junjun Deng

dengjunjun@ bit.edu.cn

1 School of Mechanical Engineering, Beijing Institute of Technology, NO. 5 Zhongguancun Street, Beijing, China

2 Collaborative Innovation Center of Electric Vehicles in Beijing, NO. 5 Zhongguancun Street, Beijing, China

\section{Introduction}

The application of wireless power transfer (WPT) technology in electric vehicles, especially the inductive power transfer (IPT) technology, effectively reduces manual interventions, making the charging process safer, more efficient and convenient. Unmanned charging enables more intelligent algorithms to be used for charging management, which improves the degree of vehicle intelligence [1-3]. The ultimate goal of autonomous vehicles is to achieve an automation level of "Level 5" (in the Society of Automotive Engineers Level of Automation scale), which corresponds to a full driving automation, but most of the current automated road vehicles remain on "Level 3", which corresponds to a conditional driving automation, or even lower levels[4]. This is largely due to technical factors, and legal factors cannot be ignored [5]. In certain areas, higher-level autonomous vehicles are already in use, such as unmanned cargo vehicles in the factory and shuttle buses in the park [6].

In the 2022 Beijing Winter Olympics, autonomous vehicles including shuttle buses, freight trucks, and road sweepers are planned to be deployed in the Shougang Park to provide convenient service. Universal wireless chargers for all 
types of autonomous vehicle become necessary. A design challenge exists due to the different chassis forms adopted by commercial vehicles and passenger cars. Electric commercial vehicles have higher chassis and heavy weight, which call for higher charging power while facing lower parking position accuracy. Therefore, the high power and interoperability are required.

In high-power IPT systems for electric vehicles, the limited onboard space is the main design constraint. The complex compensation circuit for the secondary side leads to extra onboard components, such as the double-sided inductor-capacitor-capacitor (LCC-LCC) compensation which has an extra coil and a capacitor compared with the series compensated secondary coil. The LCC-series (LCC-S) compensation topology suffers from a low system efficiency under heavy-load conditions [7]. For many high-power electric vehicle wireless charging systems, the simplest series-series (S-S) compensation topology is more suitable [8]. However, the degree of freedom of parameter adjustment of the compensation circuit is limited and the performance of the system is easily affected by the variation of the coupling coefficient, which is inevitable due to parking deviation.

To reduce the sensitivity of the coupling coefficient to parking misalignment, researchers proposed the double-D coil (DD coil) [9], which has lower longitudinal offset sensitivity and improved DD-Quadrature coil (DDQ coil) [10], and Refs [11, 12] increased the robustness of the system by increasing the size or number of primary coils. Using ground equipment with relatively loose size restrictions to enhance the coupling coefficient of the system can alleviate the problems caused by size restrictions. Another research takes parking offset into consideration [13], in which the global optimization of the coupling coefficient is performed to achieve better overall performance within the entire parking misalignment range.

When designing an IPT system for commercial vehicles, the conditions with narrow chassis and large air gap limit the application of the above methods. The fundamental flux path of the DD coil is approximately half of the coil diameter [9], which results in a limited distance for power transmission although it is insensitive to positional deviation. When used in high-power applications, the Litz wire used in the coil is thicker, resulting in a reduction in the effective number of turns of the DD coil, which further limits its application. The DDQ coil, with the same shortcomings as the DD coil, increases the volume, making it less practical in high-power applications.

As the delivered power increases, the design of the compensation topology becomes more difficult. First, the diameter of the Litz wire needs to be increased, and the ferrite needs to be thickened, so the space for the compensation circuit becomes more limited. Besides, the high voltage stress across the compensated capacitors is aggravated, which calls for high dielectric strength at high frequency. In addition, to realize the zero phase angle (ZPA) or zero voltage switching (ZVS) operation of the system in a wide range, the inductance parameters should be fine-tuned. However, under the limited freedom of the mechanical dimension, it is tricky to maintain constant values of the coupling coefficient and inductance of the magnetic coupler, especially when significant misalignments exist.

In summary, to improve the robustness of the highpower IPT system, it is necessary to optimize the design of the unipolar coil according to the characteristics of the S-S topology. After preliminary exploration, a new type of multiple series unipolar coil (MS coil) has been proposed in the conference 2021 IEEE international conference on magnetics [14].

Based on the analysis of the influence of MS coil mechanical dimension changes on electrical parameters, this paper proposes an expert model for MS coil parameter optimization, which can approach the targeted mutual inductance and self-inductance. To avoid the failure of the expert model in the optimization process and improve the optimization efficiency, the traditional simulated annealing (SA) algorithm is improved by introducing a backtracking mechanism, and the parameter combination of the MS coil is optimized using this improved algorithm. The optimization results show that compared to the traditional symmetrical coil, the misalignment sensitivity of the MS coil in the door-to-door direction of the vehicle is improved by $39.90 \%$ from $58.79 \%$ to $18.89 \%$.

The remainder of this paper is organized as follows. In Sect. 2, the selection basis and structural characteristics of the MS coil are focused. In Sect. 3, the establishment and discussion of the expert model are presented. The improvement of the simulated annealing algorithm and the combined optimization scheme are discussed in Sect. 4. In Sect. 5, the process and results of the optimized operation are introduced.

\section{Selection Basis of MS Coil}

There are four types of autonomous vehicles equipped with wireless chargers have been demonstrated in the Shougang Park for the Beijing 2022 Winter Olympics. Although their models are different, they all require a power output of $30 \mathrm{~kW}$, while the peak DC-DC efficiency is greater than or equal to $92 \%$. Therefore, the WPT system must achieve the above goals at an output voltage of 400-600 V DC. According to the introduction in Sect. 1 about the comparison of different compensation networks and the requirements for wireless charging systems for unmanned vehicles in the Winter Olympics park, the S-S compensation topology and unipolar coil scheme are selected. A typical S-S 
compensated IPT system and the S-S topology are shown in Figs. 1 and 2, where $U_{\mathrm{s}}$ and $U_{\text {ac_o }}$ are the equivalent output voltage of the inverter and the input voltage of the rectifier bridge, respectively.

In the S-S compensated IPT system, the currents of the primary and secondary coils can be given by

$\omega=\omega_{0}=1 / \sqrt{L_{1} C_{1}}=1 / \sqrt{L_{2} C_{2}}$

$I_{1}=\frac{U_{\mathrm{s}}}{R_{1}+R_{\mathrm{r}}}=\frac{R_{2} U_{\mathrm{s}}}{R_{1} R_{2}+(\omega M)^{2}}$

$I_{2}=\frac{j \omega M I_{1}}{R_{2}+R_{\mathrm{ac}}}=\frac{j \omega M U_{\mathrm{s}}}{R_{1}\left(R_{2}+R_{\mathrm{ac}}\right)+(\omega M)^{2}}$

$M=k \sqrt{L_{1} L_{2}}$

where $k$ is the coupling coefficient, and $R_{\text {ac }}$ is a simplified expression for the rectifier and the battery pack. $R_{1}$ and $R_{2}$ represent the impedance in the primary and secondary circuits when the working frequency $\omega$ is the same as the resonance frequency $\omega_{0} . R_{\mathrm{r}}$ is the reflection impedance when $\omega=\omega_{0}$. This is given by $R_{\mathrm{r}}=\frac{(\omega M)^{2}}{R_{2}+R_{\mathrm{ac}}} \cdot R_{1}$ and $R_{2}$ are small enough to be ignored.

The output current is inversely proportional to the mutual inductance. Using the equations above, the current in the primary resonant cavity can be estimated as

$I_{1} \approx \frac{R_{2} U_{\mathrm{s}}}{(\omega M)^{2}}=\frac{R_{\mathrm{ac}} I_{2}}{(\omega M)^{2}}=\frac{U_{\mathrm{ac} \_\mathrm{o}}}{j \omega k \sqrt{L_{1} L_{2}}}$

It can be seen that the voltage of the load determines the current stress of the primary side resonant circuit. Therefore, to ensure that the system can work safely under high power while considering parking misalignment, the magnetic coupler should be optimized and the coupling coefficient should be limited within a reasonable range. To compare the circular coil and a rectangular coil and decide which is more suitable for such conditions, two single-turn coil models are compared using finite element analysis (FEA). By the definition of the vehicle dynamics coordinate system, the driving direction is defined as the $X$-direction, the door-to-door direction is defined as the $Y$-direction, and the

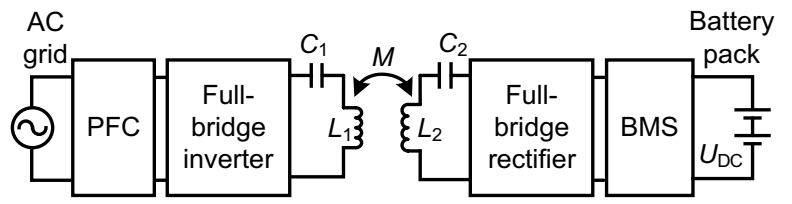

Fig. 1 Schematic of the S-S compensated IPT system

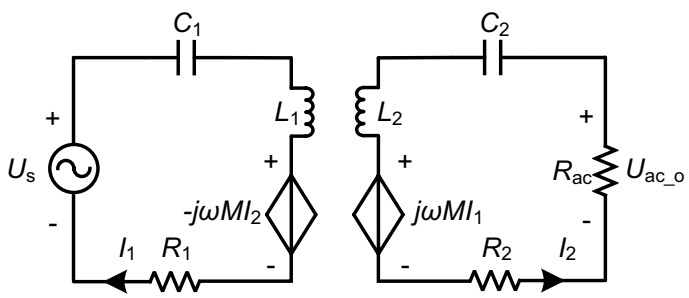

Fig. 2 The equivalent circuit of the S-S topology

upward direction perpendicular to the ground is defined as the $Z$-direction.

Because the biggest constraint of the magnetic coupler design is the size of the vehicle chassis, the diameter of the circular coil is $300 \mathrm{~mm}$ and the air gap between the primary and secondary coils is $150 \mathrm{~mm}$. The simulation results of the $X / Y$-direction misalignment sensitivity of the single-turn coils without a ferrite layer and the single-turn coil used in the simulation are shown in Fig. 3. The coupling coefficient of the circular coil and the rectangular coil all drop by more than $40 \%$ in the case of parking misalignment. This means that it is difficult to balance the design of the entire system. Simultaneously, it can be seen that the effect of the rectangular coil with higher space utilization is better, so the rectangular coil is more likely to be optimized.

Based on general experience, as long as the driver can park the vehicle accurately in the parking space, the position deviation in the $X$-direction is easier to be adjusted than that in the $Y$-direction to correct [15]. Therefore, the easiest way to realize the robustness of the coupler parameters is to balance the coupling coefficient in $Y$-direction.

In the magnetic field formed by the flat coil, the selfinductance of the primary and secondary coils will not vary significantly, regardless of misalignment conditions. Therefore, ensuring the mutual inductance limits the fluctuation

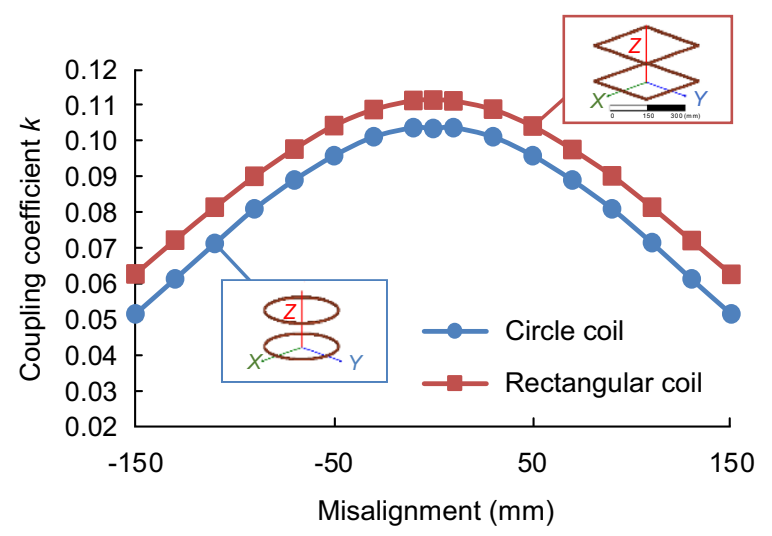

Fig. 3 FEA results of the misalignment sensitivity of circle coil and rectangular coil 
of the coupling coefficient. It can be understood from the definition of mutual inductance that the value of mutual inductance depends on the effective magnetic flux passing through the coil. In order to increase the mutual inductance in $Y$-direction, it is necessary to increase the magnetic field in $Y$-direction. The easiest way is to add additional coils in the $Y$-direction to enhance the surrounding magnetic field. There have already been many studies that have used this method. However, most of the extra coils are excited by extra supply power to achieve magnetic field enhancement in a specific area, which is a complicated solution for control.

To reduce the difficulty of circuit design and control as much as possible while realizing high robustness of the magnetic coupler, a structure with three coils connected in series is proposed, which is called MS coil. Fig. 4 gives the main design parameters and the direction of the current in the coil at a certain moment of MS coil. Among them, $l_{\text {gap }}$ refers to the gap between Litz wires, and $n_{1}$ and $n_{2}$ are the turns of the primary coil and added coils respectively. It can be seen from the direction of the current that although the MS coil has a three-coil structure, similar to a DDQ coil. But the way it forms a magnetic field is essentially different from that of a DDQ coil.

To distinguish the corner labels of the parameters conveniently, the coil in the center is numbered Coil 1, and the coils on both sides are numbered Coil 2. Fig. 4 is a top view of this coil, which means that the ferrite layer will appear below Coil 1 .

Through previous research, the effect of the MS coil in reducing the sensitivity of the misalignment of the magnetic coupler in $Y$-direction has been verified. However, the final result of the coil is given by manual screening, which lacks the necessary optimization of the parameter combination.

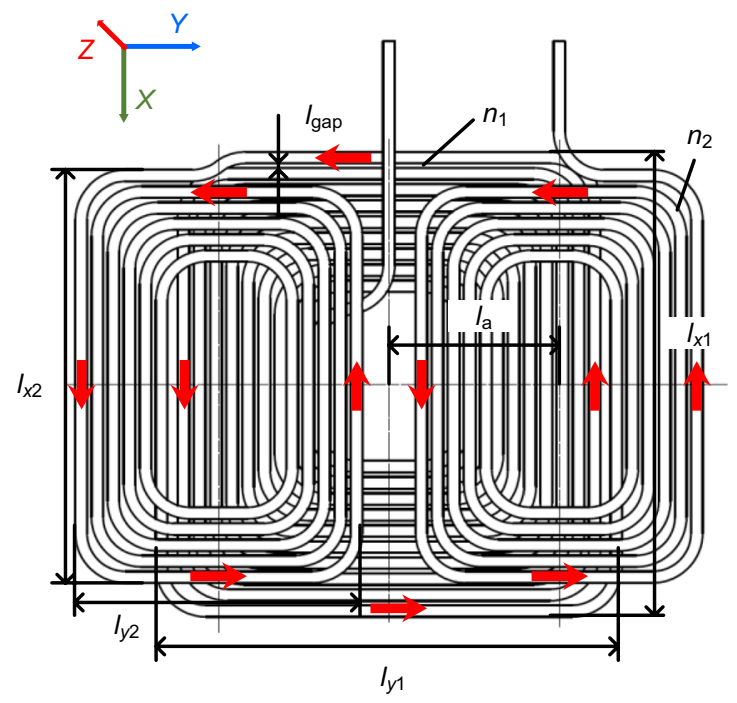

Fig. 4 Some important dimensions and current directions of MS coil
In order to obtain better design parameters of the primary coil in a limited space, a series of simulation models are built. After a deeper FEA analysis, the influence mechanism among the 8 main parameters of the MS coil is discovered.

\section{Expert Model for MS Coil Optimization}

According to the analysis in the previous sections [14], the MS coil can obtain eight degrees of freedom by adjusting its eight-parameter configuration. To get an accurate optimization guideline rather than a general description, the detailed design parameters of the IPT system demonstrated in Shougang Park should be specified.

The aim is to ensure that the four types of vehicle can be equipped the magnetic couplers with the same specification. Due to the limited chassis space, the size of the secondary coil is restricted to $380 \mathrm{~mm} \times 440 \mathrm{~mm}$, the number of turns $n_{3}=11$, and a $390 \mathrm{~mm} \times 540 \mathrm{~mm} \times 15 \mathrm{~mm}$ ferrite is placed between the coil and its aluminum shielding. At the rated output power of $30 \mathrm{~kW}$ and the output DC voltage of $400-600 \mathrm{~V}$, the maximum value of $I_{1}$ is $120 \mathrm{~A}$, and the maximum value of $I_{2}$ is $80 \mathrm{~A}$. To have the current density lower than $5 \mathrm{~A} / \mathrm{mm}^{2}, 0.1 \times 5000$ strands of Litz wire is used for the primary coil, and $0.1 \times 2500$ strands of Litz wire is used for the secondary coil. The air gap of the magnetic coupler is $150 \mathrm{~mm}$, the operating frequency of the system is $85 \mathrm{kHz}$, and the required $Y$-direction misalignment tolerance is $150 \mathrm{~mm}$.

For comparison with the proposed method, the Dagum distribution method is adopted for the primary coil's optimized design. As a result, a rectangle coil is obtained, whose dimension is $391.4 \mathrm{~mm} \times 453.2 \mathrm{~mm}$ with the number of turns $n_{1}{ }^{\prime}=9$ as shown in Fig. 5. To design the MS coil with the same secondary dimension as the rectangle coil, an FEA model is built, as shown in Fig. 6. To be noted, the ferrite for the MS coil is rotated 90 degrees to have sufficient space for the resonant capacitors and connectors installed together in the aluminum shielding of the transmitter.

For the purpose of obtaining the expert model, a series of FEA simulation are executed. Starting from a randomly

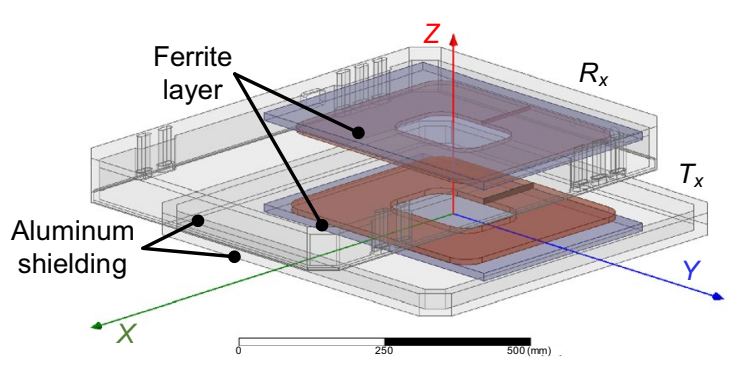

Fig. 5 Fully optimized traditional unipolar coil 


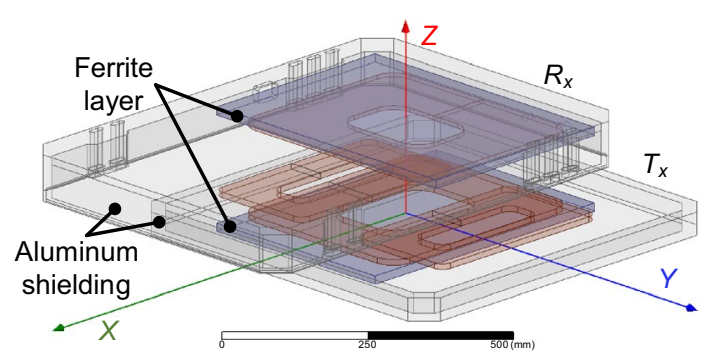

Fig. 6 FEA model of the magnetic coupler with MS coil

selected combination, the 8 dimensions of MS coil are increased in the feasible region one by one. On the premise that the three coils do not interfere with each other and the shielding, the variation range of each parameter of MS coil is shown in Group 1 of Table 1. All the simulations are performed with aligned condition and misalignments of $150 \mathrm{~mm}$ in $Y$-direction, and the results are shown in Fig. 7. Fig. $7 \mathrm{a}$ and $\mathrm{b}$ indicates how the dimensional changes affect the self-inductance of the primary coil and the coupling coefficient of the magnetic coupler, respectively. According to Fig. 7a, the self-inductance varies slightly with misalignment since the lines overlapping in two conditions. Overlapped curves can be found in Fig. 7b, which means the corresponding dimensional parameters affect the coupling coefficient approximately the same in the specific condition.

To normalize the influence of various parameters' variations on the MS coil-based coupler, the horizontal axles in Fig. 7 have been rescaled to $X^{*} . X^{*}$ is calculated as

$X^{*}=\frac{X-X_{\min }}{X_{\max }-X_{\min }}$

where $X$ represents one of the 8 parameters of the MS coil. $X_{\max }$ and $X_{\min }$, respectively, represent the upper and lower bounds of the parameters in Table 1. In Eq. (6), $X$ is calculated with its dimension, so the obtained $X^{*}$ is a dimensionless number.

Table 1 The range of FEA parameters

\begin{tabular}{lll}
\hline Symbol & Range (Group 1) & Range (Group 2) \\
\hline$l_{x 1}$ & $300-500 \mathrm{~mm}$ & $380-580 \mathrm{~mm}$ \\
$l_{y 1}$ & $300-500 \mathrm{~mm}$ & $300-500 \mathrm{~mm}$ \\
$l_{x 2}$ & $250-500 \mathrm{~mm}$ \\
$l_{y 2}$ & $350-500 \mathrm{~mm}$ & $150-190 \mathrm{~mm}$ \\
$n_{1}$ & $200-250 \mathrm{~mm}$ & $2-8$ \\
$n_{2}$ & $6-9$ & $2-4$ \\
$l_{\text {a }}$ & $4-7$ & $100-200 \mathrm{~mm}$ \\
$l_{\text {gap }}$ & $130-150 \mathrm{~mm}$ & $2-8 \mathrm{~mm}$ \\
\hline
\end{tabular}

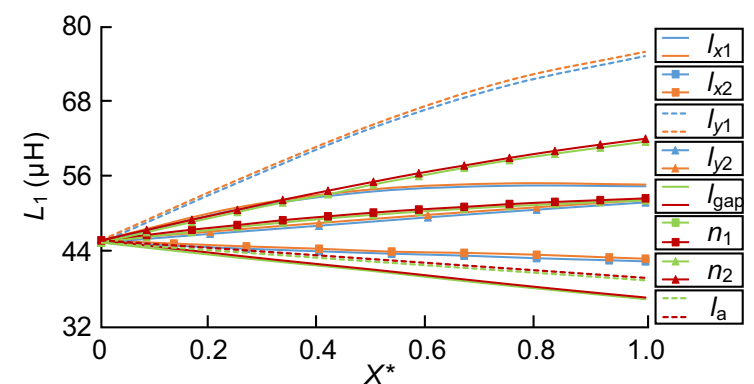

(a)

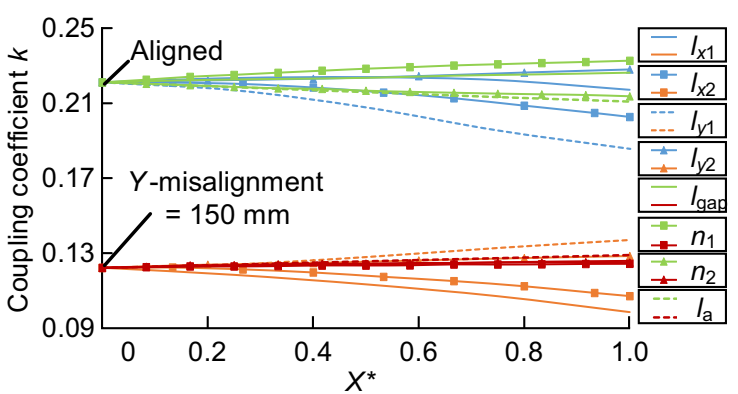

(b)

Fig. 7 Simulation results of the main parameters of the MS coil in Group 1 of Table 1

The simulation results show that most of the dimensions can be considered as linearly related to the self-inductance and the coupling coefficient, while the nonlinear ones are monotonically related to the two parameters, which can be used to build the expert model.

Moreover, it can be observed from Fig. 7b that an increase in $l_{y 1}, l_{\mathrm{x} 2}, l_{\mathrm{a}}$ and $n_{2}$ helps to achieve a more stable $k$ under the aligned and misaligned conditions.

To ensure that the experience above can be applied in a relatively wide range, simulations of another set of parameter configurations are implemented as Group 2 of Table 1. The results, which are processed in the same way as Group 1 in Table 1, are shown in Fig. 8.

By comparing Fig. 7 with Fig. 8, it can be found that although each parameter affects the performance of the magnetic coupler in different degrees due to different starting points, the overall trend is the same except for some particular parameters, such as $l_{y 1}$. In the entire simulation range, their changes are not uniform, but it can be seen that the trends near the starting point are still monotonous. For example, if the initially obtained self-inductance of the primary coil is too large, it can always be reduced by adjusting the $l_{\text {gap }}$ without changing the coupling coefficient.

The reason for this phenomenon is that the primary current through the three coils circulate in the same way. In this case, no matter how the position, size, or shape of the three coils are changed, the magnetic field formed by them will conform to the distribution form of a unipolar magnetic 


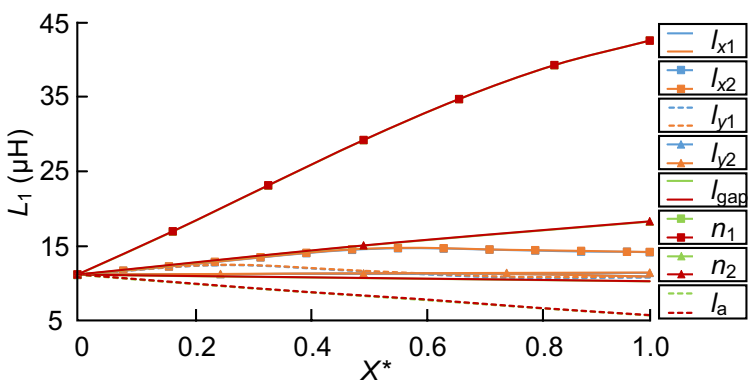

(a)

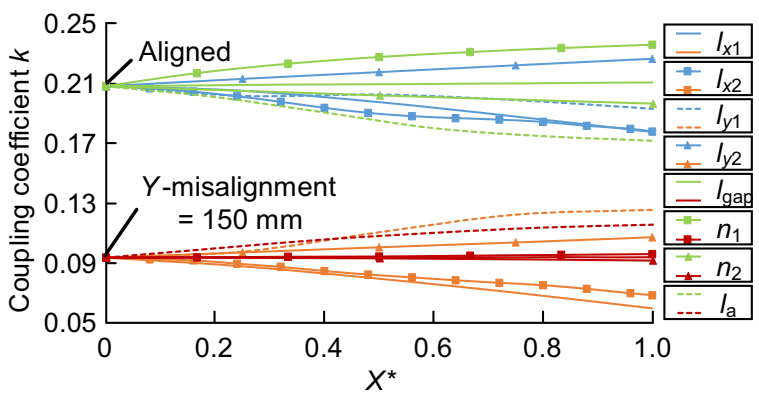

(b)

Fig. 8 Simulation results of the main parameters of the MS coil in Group 2 of Table 1

field. It can also be considered intuitively that multiple coils connected in series in the same direction have a certain magnetic field superposition.

To further explore the phenomenon of superposition, a simple simulation model is built as Fig. 9. The primary coil is composed of three coils connected in series, and their current directions are the same. Under different side lengths, the secondary coil is offset in the $Y$-direction, and the FEA results are shown in Fig. 10. Compared with single peak of coupling coefficient in Fig. 3, multi-coils placed side by side induced multi-peaks. In addition, as the width of the primary coil increases, the position of the peaks does not change, but the troughs of the coupling coefficient along $Y$-direction are strengthen by the enlarging primary coils. It is analogous to the superposition of fundamental and higher harmonics. However, this superposition is not rigorous, nor can it be expressed

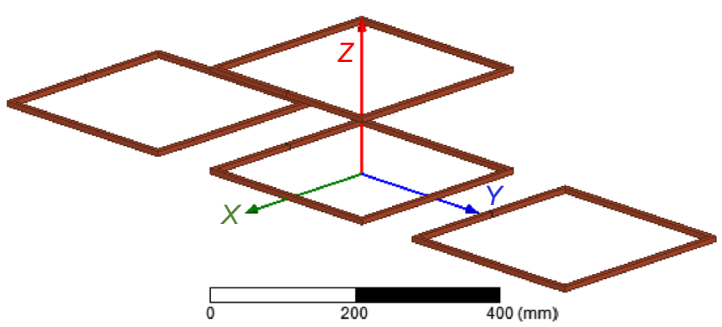

Fig. 9 FEA model used to verify the superposition of magnetic fields

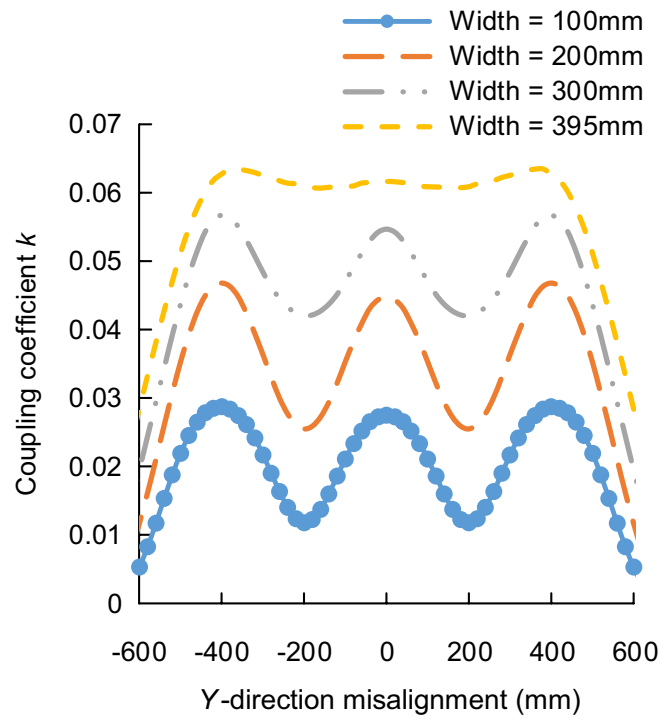

Fig. 10 The simulation results of the superimposed effect of multiple coils in series

analytically since the three coils on the primary side are coupled with each other.

In order to obtain an implementable expert model to describe the above phenomenon, some assumptions are made here:

(1) The self-inductance and the coupling coefficient vary monotonously with one of the mechanical dimensions of the MS coil when it changes in a very small range.

(2) The above monotonicity is unrelated to the starting parameter combination in the optimum searching procedure, but the increment or decrement is affected by the starting point.

(3) The influence of mechanical dimensions on electrical parameters can be superimposed in a small range.

Based on these assumptions, the following conclusions are drawn.

(1) If a larger coupling coefficient is expected, it can be achieved by increasing $n_{1}, l_{y 2}$ or $l_{\text {gap }}$, and decreasing $n_{2}$, $l_{x 1}, l_{x 2}, l_{y 1}$, or $l_{\mathrm{a}}$, vice versa.

(2) If larger self-induction is expected, it can be achieved by increasing $n_{1}, n_{2}, l_{x 1}, l_{y 1}$ or $l_{y 2}$, and decreasing $l_{x 2}$, $l_{\text {gap }}, l_{\mathrm{a}}$, vice versa.

(3) If the coupling coefficient in the misaligned case and the aligned case are expected to be closer, it can be achieved by increasing $n_{2}, l_{y 1}$, or $l_{\mathrm{a}}$, and decreasing $l_{x 1}$.

(4) The above rules (1)-(3) are consistent within a reasonably small step. 
A parameter-searching expert model can be sorted in Table 2 . In the table, $k_{\mathrm{t}}$ is the targeted coupling coefficient, $L_{1 \mathrm{t}}$ is the aimed self-inductance of the primary coil and $k_{\text {diff }}$ is the difference between the coupling coefficient in the aligned case and the misaligned case. " $\uparrow "$ means the dimensional parameter should be increased to approach the expected value of the self-inductance and coupling coefficient. Similarly, " $\downarrow$ " means the dimensional parameter should be decreased while "Null" means adjusting this parameter is invalid. It is noted when $k_{\text {diff }}$ is greater than 0 , there will be several invalid parameters. To avoid introducing too many uncertain factors into the expert model, this paper uses a two-level searching mode to identify the branch among the five situations listed in Table 2 for the next investigated parameter combination.

In some cases, the tuning direction of the parameters is determined, but contradictions exist in some other cases. For example, when the last calculation result satisfies $k<k_{\mathrm{t}}$ and $L_{1}<L_{1}, n_{1}$ should be increased for getting close to the expected values. However, when $k<k_{\mathrm{t}}$ and $L_{1}>L_{1 \mathrm{t}}$, it is not sure if $n_{1}$ should be increased. In this case, to determine the next searching direction, the parameters' variation is weighed by calculating the degree of deviation of the self-inductance and coupling coefficient from aimed values. The weighting method can be expressed as

$$
\begin{aligned}
& \operatorname{Per}_{k}=\frac{k}{k_{\mathrm{t}}} \times 100 \% \\
& \operatorname{Per}_{L_{1}}=\frac{L_{1}}{L_{1 \mathrm{t}}} \times 100 \%
\end{aligned}
$$

$P_{X}=1 \pm \frac{P e r_{k}}{P e r_{k}+\operatorname{Per}_{L_{1}}} \cdot \eta \cdot R_{(0,1)} \pm \frac{P e r_{L_{1}}}{P e r_{k}+\operatorname{Per}_{L_{1}}} \cdot \eta \cdot R_{(0,1)}$

$X^{\prime}=X \cdot P_{X}$

where $P_{X}$ is the searching factor, $X$ can be any of the eight tunning parameters of the MS coil, such as $n_{1}, n_{2}$, and so on. $\mathrm{Per}_{k}$ and $\mathrm{Per}_{L_{1}}$ are the variation percentages of coupling coefficient and self-inductance relative to their aimed values. $\eta$ is the searching step, and $R_{(0,1)}$ is a random number between 0 and 1. The value of the sign in Eq. (9) depends on the direction of the arrow in Table 2.

Therefore, the single-step parameter search process of the MS coil based on the mentioned expert model and FEA can be described in Fig. 11.

It should be noted that Fig. 11 only shows a single searching step for a new parameter combination other than the entire searching process of the optimization due to lacking the necessary conditions for looping and exiting the loop. Besides, at least two sets of simulation results are required to calculate the difference of $k_{\text {diff }}$ at two different situations.

\section{Improved SA Algorithm}

After more than half a century of development, the SA algorithm has been improved in many fields and made breakthroughs [16-18]. In the field of wireless power transfer, SA algorithms have been widely applied in the optimization of system's electrical parameters [19] and the research of output control [20]. However, the SA algorithm needs to abstract a "temperature" that is decoupled from other design parameters, so it is rarely used in coils' dimensional-parameter optimization.

The traditional SA algorithm has no directionality. Starting from the initial parameters, points are randomly selected in the entire feasible region, so that the annealing process forms a Markov chain. The degree of annealing is controlled by selecting the best point, and the current best point is discarded by probability to avoid falling into the local optimum. Therefore, the SA algorithm is effective under the conditions of a single environmental variable coupled with multiple internal variables. However, in wireless charging, the system itself does not have the property of spontaneous adjustment. Therefore, in terms of parameter optimization design, researchers often prefer using genetic algorithms or Pareto optimization to achieve multi-objective optimization [21].

Each parameter of the MS coil has hundreds of possibilities within the feasible range, using genetic algorithms, Pareto optimization, or traditional simulated annealing algorithms may waste a lot of computing power. At the same time, the feasible region of each parameter of the MS coil is strongly correlated, which is not a simple polygonal area planning problem, and the value range of the feasible region
Table 2 A parameter search expert model

\begin{tabular}{lllllllll}
\hline Situation & $n_{1}$ & $n_{2}$ & $l_{x 1}$ & $l_{x 2}$ & $l_{y 1}$ & $l_{y 2}$ & $l_{\text {a }}$ & $l_{\text {gap }}$ \\
\hline$k<k_{\mathrm{t}}$ & $\uparrow$ & $\downarrow$ & $\downarrow$ & $\downarrow$ & $\downarrow$ & $\uparrow$ & $\downarrow$ & $\uparrow$ \\
$k>k_{\mathrm{t}}$ & $\downarrow$ & $\uparrow$ & $\uparrow$ & $\uparrow$ & $\uparrow$ & $\downarrow$ & $\uparrow$ & $\downarrow$ \\
$L_{1}<L_{1 \mathrm{t}}$ & $\uparrow$ & $\uparrow$ & $\uparrow$ & $\downarrow$ & $\uparrow$ & $\uparrow$ & $\downarrow$ & $\downarrow$ \\
$L_{1}>L_{1 \mathrm{t}}$ & $\downarrow$ & $\downarrow$ & $\downarrow$ & $\uparrow$ & $\downarrow$ & $\downarrow$ & $\uparrow$ & $\uparrow$ \\
$k_{\text {diff }}>0$ & Null & $\uparrow$ & $\downarrow$ & Null & $\uparrow$ & Null & $\uparrow$ & Null \\
\hline
\end{tabular}




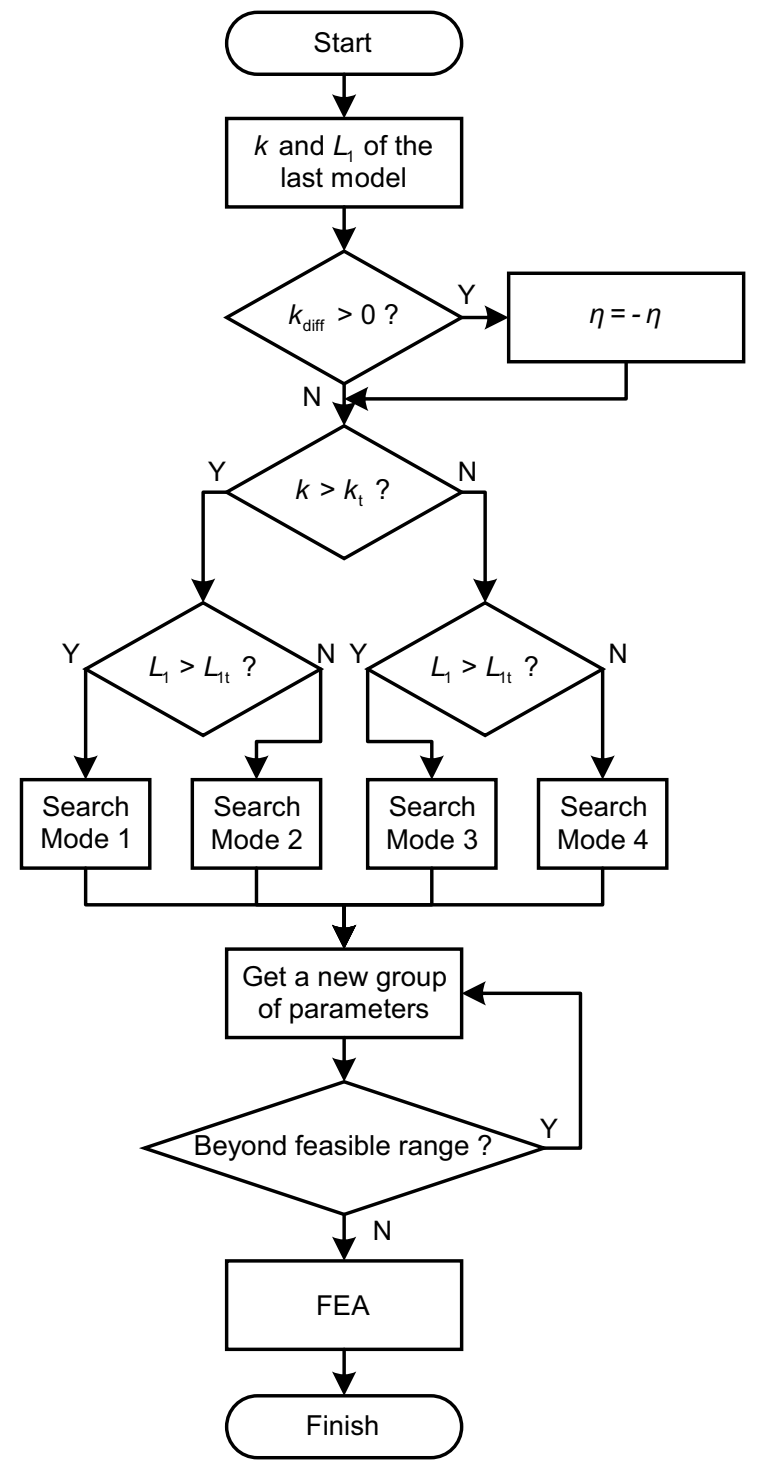

Fig. 11 Single-step parameter search process based on the expert model and FEA

cannot be given directly. However, according to the expert model established in Sect. 3, the mechanical dimensions of the MS coil can be gradually tunning to approach the targeted electrical parameter set by constructing an incremental Markov process. Therefore, combined with the expert model, the traditional simulated annealing algorithm can be improved.

The basic idea of the improved SA algorithm is as follows:

(1) In the annealing process, the evaluation function is used to select the current best point, but the searching direction is artificially given for the tunning parameters, instead of randomly selected points around the best point.
(2) The algorithm no longer probabilistically abandons the current best point but uses the backtracking mechanism to avoid falling into the local optimum, which will be described in detail below.

(3) In the optimization process, the searching step size is dynamically adjusted. The fine searching is focused on the area that close to the targeted values, and the other areas are searched roughly.

As mentioned above, the algorithm itself depends on the evaluation function to continue. Therefore, the evaluation function is the key to quickly screen out the best points while reflecting the difference in the degree of attention in the optimization process of multiple goals.

In the high-power WPT system, there is a big difference between $k$ and $L_{1}$ in terms of quantity. Therefore, when formulating the evaluation function $S$, it is necessary to normalize the coupling coefficient and self-inductance. The evaluation function selected herein is as follows:

$S=m_{1} \cdot\left|k-k_{\mathrm{t}}\right|+m_{2} \cdot\left|L_{1}-L_{1 \mathrm{t}}\right|+m_{3} \cdot\left|k_{\mathrm{mis}}-k_{\mathrm{mist}}\right|$

where $m$ is the proportionality coefficient, and $k_{\text {mis }}$ is the coupling coefficient under the misalignment condition of $150 \mathrm{~mm}$ in the $Y$-direction. By adjusting $m$, the convergence direction can be changed, and the degree of attention can be reflected. For example, in the goal of this paper, $k_{\text {mis }}$ is more important, so setting $m_{3}$ to 2.5 times of $m_{1}$ can make the point, where $k_{\text {mis }}$ is close to the targeted value of $k_{\text {mist }}$, more prominent, in other words, it will converge to $k_{\text {mist }}$ first.

Analogous to the search area adjustment mechanism of the SA algorithm, the improved SA algorithm also includes an adaptive search step. The search step can also be considered as the annealing temperature of the traditional SA algorithm. A backtracking mechanism is introduced to avoid falling into the local optimum.

The adaptive search step means that when the current searching step is completed by FEA simulation, according to the expert model, deviating from the best combination so far, a new combination of parameters will be randomly selected within a certain range for the next simulation. If the $k, L_{1}$, and $k_{\text {mis }}$ of the current best point are close to the aimed value before random selection, the step size will be reduced, and if it is far away, the step size will be enlarged.

As for the backtracking mechanism, there are three levels.

(1) First-level backtracking: There are too many random selection processes, but the points that have not been simulated in the feasible region are still not selected. This means that the model has reached the physical boundary, or there is no better solution around the current best point. The backtracking strategy in this situation is to return to the previous best point. 
(2) Second-level backtracking: The first-level backtracking is triggered multiple times during the operation. This means that several consecutive optimal points are close to the physical boundary of the model, and it is difficult to find the true global optimal point in this search direction. The backtracking strategy in this situation is to go back to the penultimate best point of the present.

(3) Three-level backtracking: The algorithm has run the optimization process many times, but has not found a better solution. This means that the algorithm may have fallen into a search for local optimal solutions. The backtracking strategy in this situation is to leave the expert model because the expert model has almost failed in the search process of the local optimal solution. After reducing the search step size, the parameters are randomly selected non-directionally near the current optimal point for simulation until the new optimal point is obtained.

If the duration of the three-level backtracking is too long, it means that there is no optimization space near the best point currently, and the algorithm will return to the previous best point to search. In the actual optimization process, the loop upper limit is set to prevent the target value from being set too large, causing the algorithm to fail to exit.

The flowchart of the entire improved SA algorithm is shown in Fig. 12. In the improved SA algorithm, every random process has a probability to select every point in the exploration area. At the same time, a set of parameter combinations can be approached from any direction. Therefore, this process conforms to the definition of the Markov process and is feasible.

\section{Simulation and Experimental Verification}

In order to verify the feasibility of the improved SA algorithm and the improvement of the robustness of the MS coil to the magnetic coupling mechanism, this paper uses the joint simulation of Maxwell and MATLAB to optimize the design of the primary coil.

The simulation environment is the same as that shown in Fig. 5. The operating frequency of the system is $85 \mathrm{kHz}$, the air gap is $150 \mathrm{~mm}$, and the Litz wire of $0.1 \times 2500$ strands is used as the secondary coil wire, and the Litz wire of $0.1 \times 5000$ strands is used as the primary wire. The external dimensions of the ferrite used are $390 \mathrm{~mm} \times 540 \mathrm{~mm} \times 15 \mathrm{~mm}$, and the material is PC40. The aluminum grade used is 1060 .

In the improved SA algorithm, the initial search step size is $10 \%$. The targeted coupling coefficient $k_{\mathrm{t}}$ is set to 0.18 , and the aimed coupling coefficient $k_{\text {mist }}$ when a $Y$-direction

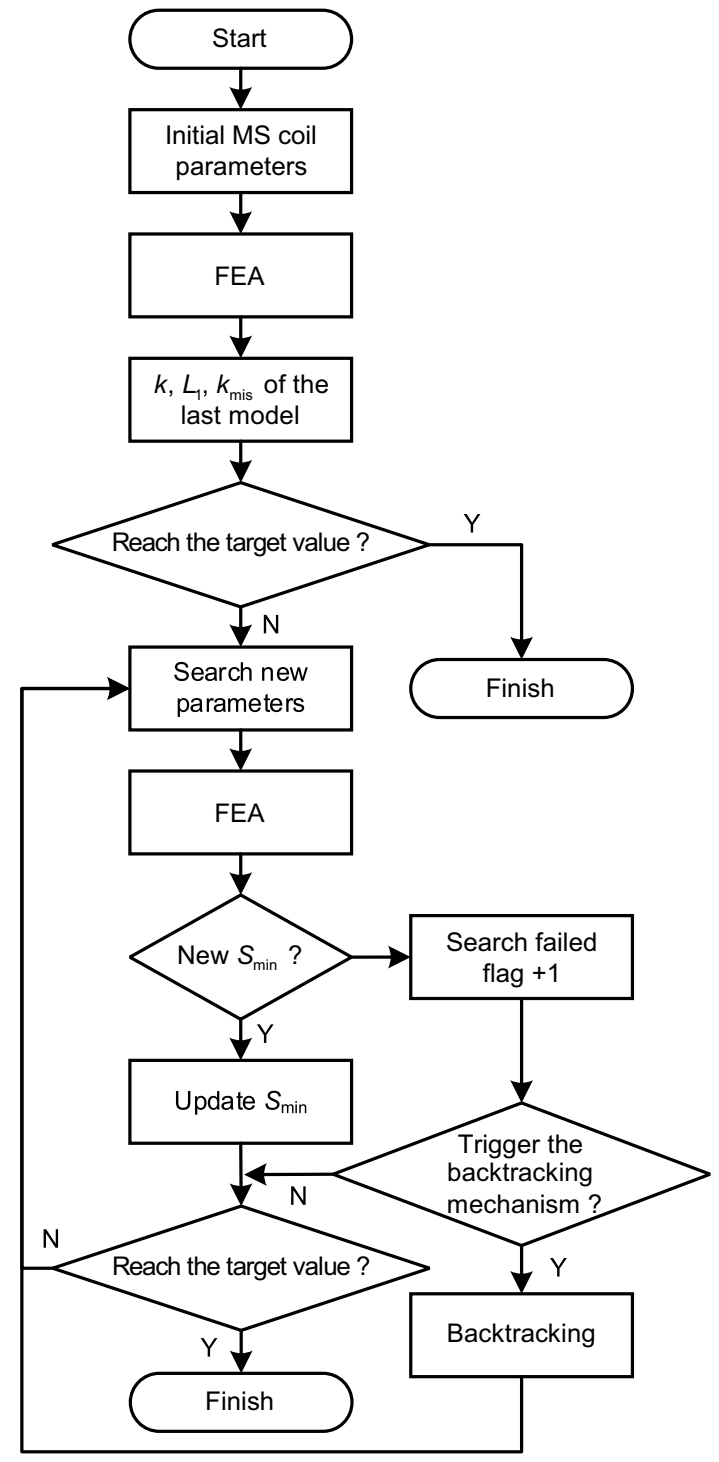

Fig. 12 Flowchart of the improved SA algorithm

misaligned $150 \mathrm{~mm}$ is set to 0.15 . The external circuit uses the CSP150 as the high-frequency resonant capacitor, whose capacitance is limited to $47.18 \mathrm{nF}$, so according to the calculation of Eq. (1), the targeted value of inductance $L_{1 \mathrm{t}}$ is set at $74.44 \mu \mathrm{H}$.

To verify that the improved SA algorithm can effectively obtain a feasible solution when extremely far away and extremely close to the targeted value, the optimization process uses two sets of initial values, some of which are shown in Table 3. To avoid excessive accumulation of simulation points near the same parameter, all parameters keep the integer part.

The result curves of the two optimization processes are shown in Figs. 13, 14, 15, 16, where $t$ is the code of the number of simulation groups, which is used to show whether the backtracking has been entered. The semi-transparent 
Table 3 Initial parameters of the improved SA algorithm

\begin{tabular}{lll}
\hline Symbol & Value (Group 1) & Value (Group 2) \\
\hline$l_{x 1}$ & $380 \mathrm{~mm}$ & $320 \mathrm{~mm}$ \\
$l_{y 1}$ & $300 \mathrm{~mm}$ & $540 \mathrm{~mm}$ \\
$l_{x 2}$ & $250 \mathrm{~mm}$ & $186 \mathrm{~mm}$ \\
$l_{y 2}$ & $150 \mathrm{~mm}$ & $229 \mathrm{~mm}$ \\
$n_{1}$ & 2 & 6 \\
$n_{2}$ & 2 & 4 \\
$l_{\text {a }}$ & $100 \mathrm{~mm}$ & $150 \mathrm{~mm}$ \\
$l_{\text {gap }}$ & $2 \mathrm{~mm}$ & $1 \mathrm{~mm}$ \\
$m_{1}$ & 200 & 200 \\
$m_{2}$ & 0.5 & 0.5 \\
$m_{3}$ & 500 & 500 \\
Operating frequency & $85 \mathrm{kHz}$ & $85 \mathrm{kHz}$ \\
Air gap & $150 \mathrm{~mm}$ & $150 \mathrm{~mm}$ \\
Litz wire & $0.1 \times 5000$ & $0.1 \times 5000$ \\
Ferrite layer & $390 \times 540 \times 15$ mm (PC40) & $390 \times 540 \times 15 \mathrm{~mm}($ PC40) \\
Aluminum shielding & Uncovered octagonal prism (1060) & Uncovered octagonal prism (1060) \\
\hline
\end{tabular}

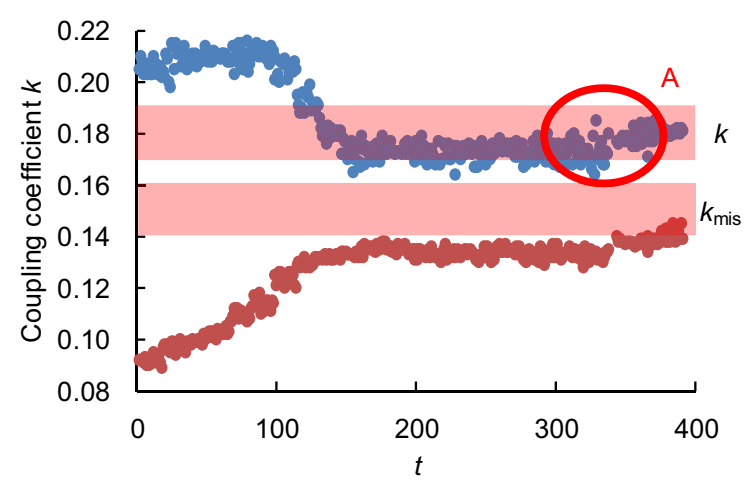

Fig. 13 The optimized progress of the coupling coefficient of Group 1 in Table 3

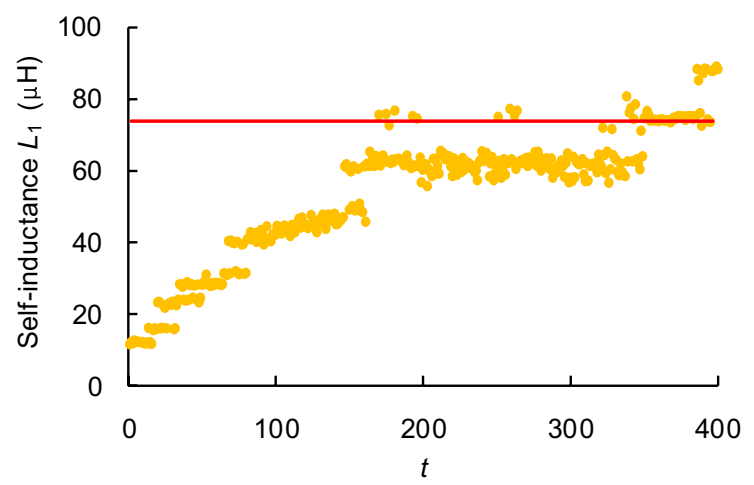

Fig. 14 The optimized progress of the self-inductance of Group 2 in Table 3

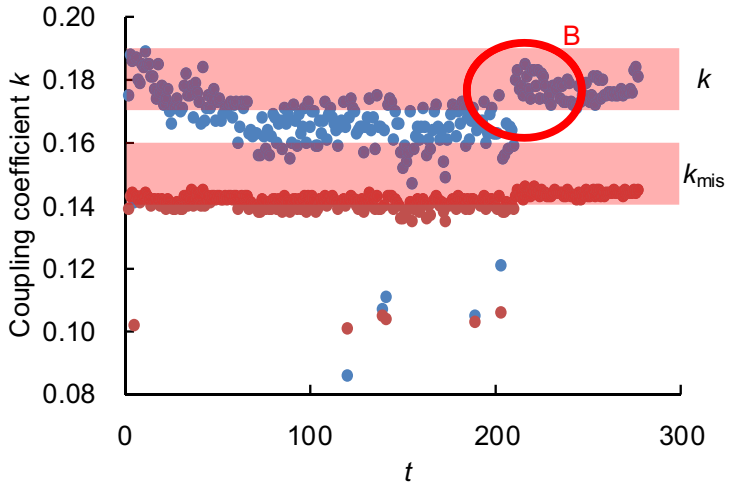

Fig. 15 The optimized progress of the coupling coefficient of Group 2 in Table 3

red band-shaped area in the figure is the allowable range of the target performance parameters. Since this area is very narrow in self-inductance, it looks like a line. It can be seen from the figure that whether it is a point that is very far from the optimization target or a relatively close point, the improved SA algorithm can guide the optimization process to the final target. The improved SA algorithm ensures that the convergence rate of the target solution is controlled between 300 and 400 simulations. At the same time, due to the parallel computing function of Maxwell, under the limited computing power of the laboratory, 16 simulation points are calculated during each optimization process. Therefore, the actual iterations are between 20 and 30 times. Lenovo P710 workstation (CPU: Intel(R) Xeon(R) E5-2640 v4 @ 2.40 GHz; RAM: 64.0 GB; SSD: Western Digital AN1500 $1 \mathrm{~TB}$ ) is used. The time for the optimization is about $10-12 \mathrm{~h}$. 


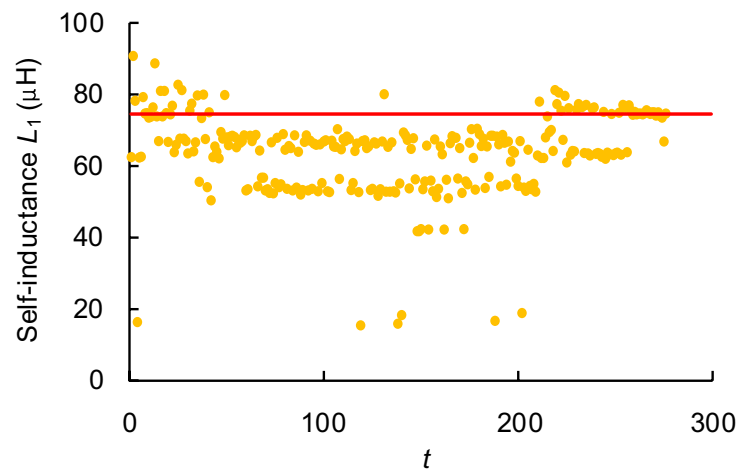

Fig. 16 The optimized progress of the self-inductance of Group 1 in Table 3

There are some discontinuities in the results of Figs. 13, 14, 15 and 16. For example, there are obvious breaks in areas A and B in Figs. 13 and 15, which means that the backtracking mechanism is triggered here. According to the observation of other simulation parameters, area A triggered the first-level backtracking, and area B triggered the thirdlevel backtracking. The layer structure of self-inductance in Figs. 14 and 16 is due to the discontinuity of parameters, especially $n_{1}$ and $n_{2}$.

According to the optimization results, the optimal parameter combination and the performance parameters of the corresponding MS coil magnetic coupler are shown in Table 4. The magnetic coupler optimized by the improved SA algorithm is shown in Fig. 17.

It can be seen from Fig. 18 that without changing any external conditions, the coupling coefficient fluctuation of the magnetic coupler in the $Y$-direction is reduced from $58.79 \%$ of the fully optimized traditional coil to $18.89 \%$. Thus, the misalignment sensitivity of the magnetic coupler in the $Y$-direction is reduced by $40 \%$. The legend in the figure, the fully optimized traditional coil, refers to the asymmetric coil structure optimized according to the Dagum distribution in Refs. [11] and [13].

Table 4 Initial parameters of the improved SA algorithm

\begin{tabular}{ll}
\hline Symbol & Value \\
\hline$l_{x 1}$ & $374 \mathrm{~mm}$ \\
$l_{y 1}$ & $568 \mathrm{~mm}$ \\
$l_{x 2}$ & $193 \mathrm{~mm}$ \\
$l_{y 2}$ & $236 \mathrm{~mm}$ \\
$n_{1}$ & 7 \\
$n_{2}$ & 4 \\
$l_{\mathrm{a}}$ & $158 \mathrm{~mm}$ \\
$l_{\text {gap }}$ & $1 \mathrm{~mm}$ \\
$k$ & 0.180 \\
$L_{1}$ & $74.51 \mu \mathrm{H}$ \\
$k_{\text {mis }}$ & 0.146 \\
\hline
\end{tabular}

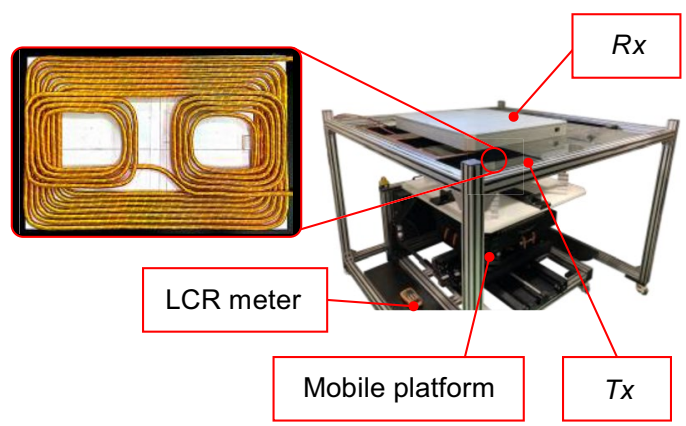

Fig. 17 The laboratory prototype and test platform

According to the analysis in Sect. 2, the reduction of the fluctuation of the coupling coefficient means that in the application of high-power wireless charging, the vehicle can park more freely. When the vehicle is parked accurately and the $Y$-direction misalignment occurs, a more stable coupling coefficient is conducive to configuring the system parameters at the beginning of the design. In this way, large reactive power will not appear in the transmit coil due to the sudden decrease in the coupling coefficient, which will increase the switching stress of the inverter.

Based on the optimized parameters in Table 4, a laboratory prototype of a magnetic coupler is built to verify the accuracy of the entire optimal design method. The photograph of the transmitting coil and the test bench is shown in Fig. 17.

The self-inductance and mutual inductance of the coil are measured using Keysight's U1733C LCR meter. The mobile platform can simulate an offset of plus or minus $150 \mathrm{~mm}$ in the $Y$-direction. The measured self-inductance and coupling coefficient of the optimized MS coil is shown in Fig. 19a and $b$, respectively. The solid line is the simulation result, and the points are the actual measurement results. From the results shown in Fig. 19, the maximum deviation of the coupling coefficient is about 0.02 , and the maximum deviation of the self-inductance is about $1.5 \mu \mathrm{H}$. The maximum coupling coefficient deviation between the simulated data and the measured data is $11.11 \%$, and the maximum self-inductance deviation is $1.96 \%$, which is inevitable and acceptable in the process of assembling the prototypes.

First of all, there are connecting wires between the three unipolar coils and between the coil and external circuits, which leads to an increase in the amount of Litz wire used in the actual prototype of the MS coil compared with the simulation model. Therefore, the self-inductance in the measured results is a bit larger and the coupling coefficient is a bit smaller than those in FEA model. Besides, in the process of testing and connecting the Litz wire of coils, an additional inductance will inevitably be added to the coil loop, resulting in a deviation of the total self-inductance value. As for 


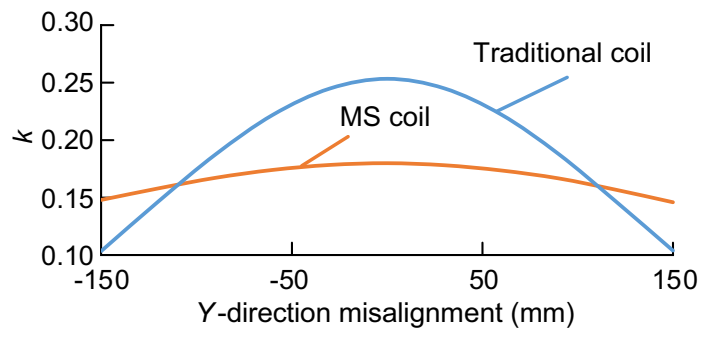

Fig. 18 The misalignment sensitivity of the MS coil optimized by the improved SA algorithm compared with that of the fully optimized traditional coil

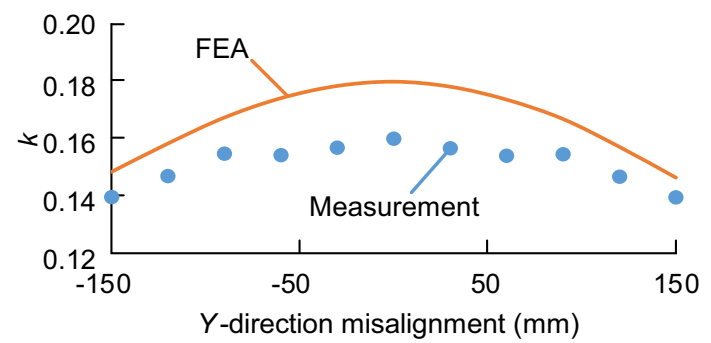

(a) Coupling coefficient

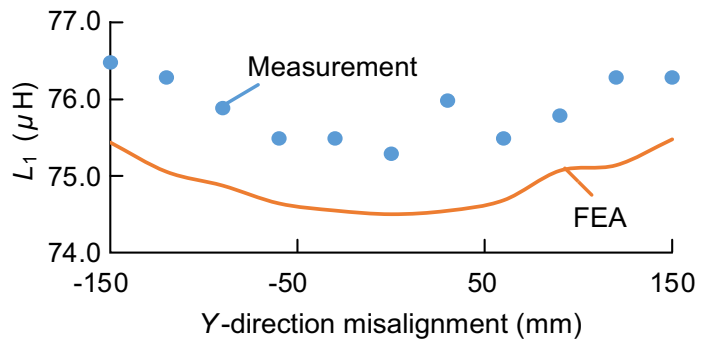

(b) Self-inductance

Fig. 19 Measurement results

the left and right not being completely symmetrical, the reason is that the aluminum shielding shell is not completely symmetrical. In summary, the accuracy of the simulation results can still be accepted.

According to the results in Fig. 19, the optimized MS coil can reduce the variation of the coupling coefficient to $12.89 \%$ in the case of $Y$-direction offset. In summary, the improved SA algorithm is proven to be feasible in the application of MS coil parameter combination optimization and the FEA analysis is accurate.

\section{Conclusions}

To improve the misalignment tolerance of high-power wireless chargers for unmanned electric trucks and electric buses, the design of the magnetic coupler is optimized, and a new type of MS coil structure is presented. Through comparative analysis by FEA, an expert model is built while an improved $\mathrm{SA}$ algorithm is proposed. By using the improved SA algorithm for optimization, the MS coil has a lower misalignment sensitivity in the door-to-door direction than the fully optimized asymmetric unipolar coil with the same inductance parameters, which reduces the current stress under the misalignment conditions caused by parking deviation. Through the joint simulation of the improved SA algorithm, the reliability of the expert model has been verified and the parameters of the MS coil are optimized. Finally, the primary coil holds a coupling coefficient of 0.180 for aligned condition, a coupling coefficient of 0.146 for $150 \mathrm{~mm}$ misaligned in $Y$-direction and a self-inductance of 74.51 $\mu \mathrm{H}$, which means the misalignment sensitivity is reduced to $18.89 \%$. A wireless charging system equipped with an optimized MS coil was built, and its measured DC-DC efficiency exceeded $93 \%$. However, this paper only considers the system stability, without further analysis of efficiency and economy. An optimized design that considers more objectives will be carried out in the future.

Acknowledgements This work was supported by the National Key Research and Development Program of China (No. 2019YFE0104700)

\section{Declarations}

Conflict of interest On behalf of all the authors, the corresponding author states that there is no conflict of interest.

Open Access This article is licensed under a Creative Commons Attribution 4.0 International License, which permits use, sharing, adaptation, distribution and reproduction in any medium or format, as long as you give appropriate credit to the original author(s) and the source, provide a link to the Creative Commons licence, and indicate if changes were made. The images or other third party material in this article are included in the article's Creative Commons licence, unless indicated otherwise in a credit line to the material. If material is not included in the article's Creative Commons licence and your intended use is not permitted by statutory regulation or exceeds the permitted use, you will need to obtain permission directly from the copyright holder. To view a copy of this licence, visit http://creativecommons.org/licenses/by/4.0/.

\section{References}

1. Li, S., Mi, C.C.: Wireless power transfer for electric vehicle applications. IEEE. J. Emerg. Sel. Topics Power Electron. 3, 4-17 (2015). https://doi.org/10.1109/JESTPE.2014.2319453

2. Schofer, J.L., Mahmassani, H.S.: A vision for transportation infrastructure. Mobil. 2050 (2016)

3. Guidi, G., Lekkas, A.M., Stranden, J.E., Suul, J.A.: Dynamic wireless charging of autonomous vehicles: small-scale demonstration of inductive power transfer as an enabling technology for self-sufficient energy supply. IEEE Electrification Mag. 8, 37-48 (2020). https://doi.org/10.1109/MELE.2019.2962888

4. National highway traffic safety administration: 2016 update to preliminary statement of policy concerning automated vehicles, 
https://www.nhtsa.gov/document/2016-update-preliminary-state ment-policy-concerning-automated-vehicles (2016). Accessed 15 May 2021

5. Dolianitis, A., Chalkiadakis, C., Mylonas, C., Tzanis, D.: How will autonomous vehicles operate in an unlawful environment? The potential of autonomous vehicles for disregarding the law. Paper presented at the 2019 6th international conference on models and technologies for intelligent transportation systems (MTITS), Cracow University of Technology, Poland, 5-7 June 2019.

6. Systems Control Technology, I., Palo, A.: Roadway powered electric vehicle project track construction and testing program phase 3d. UC Berkeley: California Partners for Advanced Transportation Technology (1994)

7. Chen, Y., Zhang, H., Shin, C., Seo, K., Park, S., Kim, D.: A comparative study of S-S and LCC-S compensation topology of inductive power transfer systems for ev chargers. Paper presented at the 2019 IEEE 10th international symposium on power electronics for distributed generation systems (PEDG). Xi'an, China, 3-6 June 2019

8. Bosshard, R., Iruretagoyena, U., Kolar, J.W.: Comprehensive evaluation of rectangular and double-D coil geometry for $50 \mathrm{~kW} / 85$ $\mathrm{kHz}$ IPT system. IEEE J. Emerg. Sel. Topics Power Electron. 4, 1406-1415 (2016). https://doi.org/10.1109/JESTPE.2016.26001 62

9. Budhia, M., Covic, G.A., Boys, J.T., Huang, C.Y.: Development and evaluation of single sided flux couplers for contactless electric vehicle charging. Paper presented at the 2011 IEEE energy conversion congress and exposition, Phoenix, AZ, USA, 17-22 Sep. 2011

10. Budhia, M., Boys, J.T., Covic, G.A., Huang, C.: Development of a single-sided flux magnetic coupler for electric vehicle IPT charging systems. IEEE Trans. Ind. Electron. 60, 318-328 (2013). https://doi.org/10.1109/TIE.2011.2179274

11. Qu, X., Jing, Y., Han, H., Wong, S.-C., Tse, C.K.: Higher order compensation for inductive-power-transfer converters with constant-voltage or constant-current output combating transformer parameter constraints. IEEE Trans. Power Electron. 32, 394-405 (2017). https://doi.org/10.1109/TPEL.2016.2535376

12. He, R., Ning, G., Jiang, Y., Zhao, K., Fu, M.: A D4Q pad with high misalignment tolerance for inductive power transfer system. Paper presented at the 2020 IEEE PELS workshop on emerging technologies: wireless power transfer (WoW), Seoul, Korea, 15-19 Nov. 2020

13. Li, L., Wang, Z., Gao, F., Deng, J., Wang, S.: FEA-assisted optimization design of asymmetric DD type structure magnetic coupler for wireless electric vehicle charger. Paper presented at the 2020 IEEE 29th international symposium on industrial electronics (ISIE), Delft, Netherlands, 17-19 June 2020

14. Li, L., Wang, Z., Feng, Z., Wang, S., Deng, J., Dorrell, D.: The electric vehicle wireless charging application oriented coupler robust optimization design with multiple series unipolar coils. Paper presented at the INTERMAG conference 2021. Underline Science Inc. (2021)

15. Birrell, S.A., Wilson, D., Yang, C.P., Dhadyalla, G., Jennings, P.: How driver behaviour and parking alignment affects inductive charging systems for electric vehicles. Transp. Res. Part C: Emerg. Technol. 58, 721-731 (2015). https://doi.org/10.1016/j.trc.2015.04.011

16. Lee, S., Kim, S.B.: Parallel simulated annealing with a greedy algorithm for bayesian network structure learning. IEEE Trans. Knowl. Data Eng. 32, 1157-1166 (2020). https://doi.org/10.1109/ TKDE.2019.2899096
17. Li, C., You, F., Yao, T., Wang, J., Shi, W., Peng, J., He, S.: Simulated annealing particle swarm optimization for high-efficiency power amplifier design. IEEE Trans. Microw. Theory Tech. 69, 2494-2505 (2021). https://doi.org/10.1109/TMTT.2021.3061547

18. Zhan, S.H., Zhang, Z.J., Wang, L.J., Zhong, Y.W.: List-based simulated annealing algorithm with hybrid greedy repair and optimization operator for 0-1 knapsack problem. IEEE Access. 6, 54447-54458 (2018). https://doi.org/10.1109/ACCESS.2018. 2872533

19. Lu, S., Zuo, C., Piao, C.: The parameters optimization of MCRWPT system based on the improved genetic simulated annealing algorithm. Math. Probl. Eng. 2015, 1-10 (2015). https://doi.org/ $10.1155 / 2015 / 174868$

20. Lin, Z., Wang, J., Fang, Z., Hu, M., Cai, C., Zhang, J.: Accurate maximum power tracking of wireless power transfer system based on simulated annealing algorithm. IEEE Access. 6, 60881-60890 (2018). https://doi.org/10.1109/ACCESS.2018.2876470

21. Deng, J., Zhang, Y., Wang, S., Wang, Z., Yang, Y.: The Design and coupler optimization of a single-transmitter coupled multireceiver inductive power transfer system for maglev trains. IEEE Trans. Transp. Electrific. (2021). https://doi.org/10.1109/tte.2021. 3061156

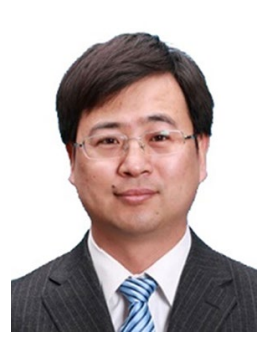

Zhenpo Wang received the Ph.D. degree in Automotive Engineering from Beijing Institute of Technology, Beijing, China, in 2005. $\mathrm{He}$ is currently a professor with Beijing Institute of Technology, the associate director of Collaborative Innovation Center for Electric Vehicles in Beijing and the director of National Engineering Laboratory for Electric Vehicles. His current research interests include the pure electric vehicle integration, packaging and energy management of battery system and big data analysis. Prof. Zhenpo Wang has been the recipient of numerous awards including the Second Prize of National Science and Technology Progress.

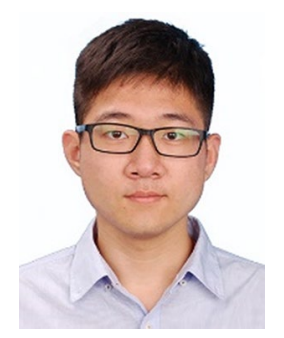

Lantian Li received the B.S. degree in Mechanical Engineering from Beijing Institute of Technology, Beijing, China, in 2017. He is studying for a Ph.D. degree in mechanical engineering in the National Engineering Laboratory for Electric Vehicles from Beijing Institute of Technology, China and the Collaborative Innovation Center of Electric Vehicles in Beijing, China. His main research interests include the optimization of magnetic coupler and dynamic wireless power transfer.

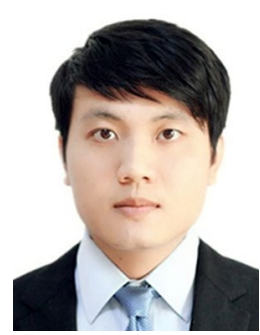

Junjun Deng received his B.S., M.S. and Ph.D. degrees in Electrical Engineering from Northwestern Polytechnical University, Xi' an, China, in 2008, 2011 and 2015, respectively. From 2011 to 2014, he was a visiting scholar with the Department of Electrical and Computer Engineer, University of Michigan, Dearborn. In 2016, he joined the Faculty of Vehicle Engineering, Beijing Institute of Technology. His research interests include wireless power transfer, resonant power conversion, and electric motor drive for electric vehicles. 


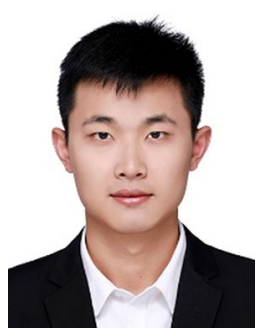

Baokun Zhang received his B.S. degree in Vehicle Engineering from Beijing Institute of Technology, Beijing, China, in 2019. He is currently pursuing the Ph.D. degree in Mechanical Engineering with the National Engineering Laboratory for Electric Vehicles, at Beijing Institute of Technology. His research interests include thermal design of IPT systems and bidirectional wireless power transfer.

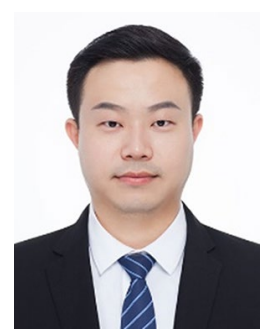

Shuo Wang received the B.S. from Shandong University of Science and Technology, Qingdao, China, M.S. from China University of Mining and Technology, Beijing, China, and $\mathrm{Ph} . \mathrm{D}$ degree in Engineering from the Faculty of Engineering and Information Technology (FEIT), University of Technology Sydney in 2017. He is assistant professor at Beijing Institute of Technology, Beijing. He was a postdoctoral research fellow with the Beijing Institute of Technology (2017-2021). His research interests include wireless charging, electric vehicle and its big data analysis. 